\title{
Humanistic Management of Social Innovation in Service (SIS): an Interdisciplinary Framework
}

\author{
Sertan Kabadayi ${ }^{1} \cdot$ Linda Alkire (née Nasr) $^{2} \cdot$ Garrett M. Broad $^{3}$. \\ Reut Livne-Tarandach ${ }^{4} \cdot$ David Wasieleski $^{5,6} \cdot$ Ann Marie Puente $^{7}$
}

Received: 23 April 2019 / Accepted: 1 July 2019 / Published online: 16 July 2019

(C) Springer Nature Switzerland AG 2019

\begin{abstract}
Humanistic Management and Transformative Service Research literatures share the common goal of addressing the increasingly growing global challenges faced by humanity. Recently, organizations have been called to further engage in social innovation in service (SIS) in an attempt to address these challenges. However, the existing service literature does not offer explicit processes regarding how to manage these social innovation efforts at the human interaction level. By drawing on both Humanistic Management and Service literatures, this paper develops a conceptual framework to guide the social innovation in service efforts. More specifically, this paper aims to answer a key question of: how can organizations manage human interactions to help maximize social innovation in service (SIS) outcomes? This paper identifies four foundational values (respect, trust, fairness, and inclusion) that should be at the core of the proposed processes (communication, collaboration, and compassion) needed in order to achieve the desired outcomes of SIS (relieving suffering, increasing well-being, and protecting and promoting human dignity). Subsequently, a typology of service organizations is offered with different combinations of processes at the human interaction level, highlighting the synergistic effect of the three identified processes. The proposed framework in this paper is a first step in bridging two disciplines to highlight their potential and role in addressing the global challenges.
\end{abstract}

Keywords Social innovation in services (SIS) - Transformative service research · Service encounter $\cdot$ Human interactions · Compassion · Communication · Collaboration · Dignity · Wellbeing

\section{Introduction}

Our world is facing a wide range of problems, from poverty and hunger, to lack of access to clean water and quality education, to an ever growing refugee population, and spreading forms

Sertan Kabadayi

kabadayi@ fordham.edu

Extended author information available on the last page of the article 
of inequality (United Nations 2018). The size and complexity of these problems require a holistic approach for addressing them, involving participation from a broad group of actors (Aksoy et al. 2019) and integration of knowledge from various research disciplines (Gustafsson et al. 2016). Two such useful disciplines could be that of service research and humanistic management.

First, service research is essential because many of those problems are service related since they result from failures in major service sectors such as healthcare, education, government, and knowledge-based industries. Recently, the concept of Social Innovation in Service (SIS), defined as "the creation of novel, scalable, and sustainable market-based service offerings that solve systemic societal problems," (Aksoy et al. 2019) has been proposed to highlight the role of services in finding solutions to the global problems mentioned above. Nevertheless, the success of SIS efforts is conditional upon recognizing that at the core of services is the human interaction. The current service literature does not offer explicit processes regarding how to manage human interaction for SIS purposes. To address this gap, we turn to the Humanistic Management literature to identify such processes.

Humanistic Management has been developed as a distinct perspective on management where the focus is on the human being and underlying dynamics of care and dignity (Pirson 2017a). It aims to create a more balanced relationship between objects that can be exchanged in markets as well as less tangible items related to promoting the flourishing of human beings (Pirson 2017b). Therefore, humanistic management as a discipline highlights who we are as human beings, as at the heart of this humanistic management approach lies a concern for the respect for the dignity and humanity of employees, customers, and anyone else affected by the company's actions (Pirson 2017a). While the extant humanistic management literature has focused on how organizations can be structured and managed to maximize outcomes that would benefit individuals and communities, the analysis and procedures at the human interaction level have received little attention. Service research could help address this gap by introducing its focus on the human interaction as a level of analysis.

This paper offers a conceptual framework that draws on both Humanistic Management and Service literatures to answer a key question: "how can organizations manage human interactions to help maximize social innovation in service (SIS) outcomes?" While different enablers have been identified at micro, meso, and macro levels to help SIS implementation (Aksoy et al. 2019), the processes through which human interactions can be managed between company employees' and customers', among employees themselves, and between customers themselves, may increase the effectiveness of such efforts and therefore would help service organizations succeed in their social innovation endeavors. More specifically, this paper makes three contributions. First, the framework introduced in this paper adds value to the existing humanistic management literature by emphasizing the importance of focusing on human interactions in its approach. Second, this paper proposes a typology of service organizations with different combinations of humanistic management processes at the human interaction level (i.e. communication, collaboration, and compassion), that can be used by service organizations to enhance their social innovation efforts. This proposed typology suggests that social innovation efforts would be most effective in producing intended outcomes when all three processes are simultaneously present in a service organization. However, when one of the processes is absent, then the outcome is suboptimal. Finally, this paper and the proposed typology offer a first step in bridging two disciplines, i.e. humanistic management and service research, to highlight their potential and role in addressing global challenges. 
This paper is organized as follows: First, literature on human interaction in services and social innovation in services is reviewed. Subsequently, four underlying core values of humanistic organizations, i.e. respect, trust, fairness, and inclusion, are introduced and defined. Then, a theoretical framework consisting of three processes (i.e. communication, collaboration and compassion) is proposed. Outcomes borrowed from both Humanistic Management and Transformative Service Research (TSR) (i.e. reducing suffering, uplifting well-being and protecting and promoting human dignity) are merged and explained as they relate to social innovation efforts of service organizations. Afterwards, four typologies are synthesized and the resulting possible outcomes of each typology are discussed. Potential challenges and obstacles to a successful implementation of the framework are discussed. Finally, theoretical and managerial implications of the typologies are presented and future research ideas are offered.

\section{Services and the Human Interaction Level}

At the core of services is the concept of "service encounters." Service encounters are defined as "human interactions" or dyadic exchanges involving service customers and providers (Solomon et al. 1985). In fact, the Service Triangle concept developed by Grönroos (1998) visualizes the various human interactions taking place within an organization. The three interlinked actors create three dyads (employee-firm (EF), customer-firm (CF) and customeremployee (CE) working together to co-create services. Furthermore, customers interact with other customers (both offline and online) as well as employees interact with each other within teams and departments creating further dyads of customer to customer (CC) interactions and employee to employee (EE) interactions. All those service encounters can be labeled "social exchanges" (Patterson et al. 2006), wherein the norms, roles, and expectations of both service employees and customers are influenced by each party's personal and cultural setting.

Human encounters have traditionally received the most attention from service researchers and practitioners given that they are at the core of what constitutes a service and are essential for a service success. Research suggests that for customers, front-line employees represent the core of the company and therefore they look for exchanges wherein they can feel comfortable (Lloyd and Luk 2011), engaged (Brodie et al. 2011), build trust (Gabbott and Hogg 2001; Sharma and Patterson 1999), and develop rapport (Medler-Liraz 2016; Gutek et al. 2002). This connection with the front-line employee is found to be detrimental in the customer's evaluation of the service outcome (Van Dolen et al. 2004). For the front-line employees, customers could be a major source of motivation and emotional labor (Grandey 2000). As such, service researchers examined the impact of customer interaction on employee productivity and wellbeing (e.g., Nasr et al. 2015). Finally, service companies who are striving for success realize the importance of investing in service encounters by working on decreasing stress levels (Bailey et al. 2001), strengthening the relationships (Verhoef 2003), and increasing positive emotions between front-line employees and customers as a way to build positive service experiences (Gentile et al. 2007).

Another more recent concept that emphasizes the importance of human interaction in a service encounter is "value co-creation." Service provision and service research rely on the premise that value is co-created when individuals integrate their resources in a mutually beneficial way during a service encounter (e.g., Vargo et al. 2008). In other words, value cocreation is an outcome of an ongoing process of multiple interactions and multidirectional resource integration where all individuals collaboratively create value (Vargo and Lusch 2008). 
Despite the tremendous work done to improve the human interaction quality in services, most of the service research to date has ignored possible resource asymmetry as part of the value co-creation process, such that one of the parties to the exchange is dependent or oppressed and is not an equal contributor/beneficiary to the exchange (Fisk et al. 2018). This leads to "Actor to Actor" exchanges, characterized by one-way interaction rather than a true co-creation. Fisk et al. (2018) stress the need for collaboration between actors and more inclusive practices whereas the exchange becomes "Actor with Actor" that leads to co-creation of value in its best way. Authentic co-creation service encounters move beyond the possibility of a service provider demonstrating power over a service recipient, and instead demonstrate a service provider having power with a service receiver (Miller and Stiver 1997).

\section{Social Innovation in Service}

Aksoy et al. (2019) propose a transdisciplinary framework for guiding Social Innovation in Service (SIS) to create "novel, scalable and sustainable" offerings by service organizations as potential solutions to systemic global problems. As such, they were among the first to bridge the disciplines of "social innovation" and "service research." In fact, this idea of SIS is built on the Transformative Service Research (TSR) framework within the services field. Based on the premise that services fundamentally affect human lives and well-being (Anderson and Ostrom 2015), Transformative Service Research (TSR) was introduced as service research that seeks to improve the well-being outcomes of consumer entities including individuals, collectives, and ecosystems (Anderson et al. 2013; Ostrom et al. 2010). As such, TSR highlights the role of services in uplifting the well-being of individual and societal actors during the interactions between service (e.g., service organizations and employees) and consumer entities (e.g., individuals, collectives) (Anderson et al. 2013). Therefore, the role of services for social innovation purposes is nicely aligned with the existing TSR literature.

Social innovation, as a field, has been gaining substantial attention in the recent years (van der Have and Rubalcaba 2016). This popularity is mainly due to the rising interest in creating multi-stakeholder solutions to social issues and problems. Ashoka (2019) defines social innovation as, "A methodology to create social value (and potentially economic value) at a systems change level, which addresses the root of a problem." Aksoy et al. (2019) argue, that in the face of the growing global challenges, service firms and researchers are well equipped to address the rising uncertainty of the future. Subsequently, they discuss micro, meso, and macro level actors and enablers in the service firm ecosystem that can help bring about SIS.

Aksoy et al. (2019) advise that micro level actors range from front-line employees to Clevels executives who, by possessing enablers such as psychological capital, risk taking, and compassion, can facilitate SIS implementation. Similarly, the meso level depicts the service firm whose characteristics and processes such as organizational culture, institution elements, leadership and empowerment are the catalyst for SIS. Finally, the macro level actors consist of external organizations or groups interacting with the service firm (e.g., communities, nongovernmental organizations (NGOs) and governments) and as such can be enabled by economic factors, government policy, and technology to achieve SIS. This multilevel approach is in line with the latest work within the service ecosystem literature (Akaka et al. 2017), stressing level embeddedness as well as the need for collaboration between levels.

Building upon the SIS framework of Aksoy et al. (2019), we propose that SIS success is contingent upon the success of human interaction at the micro level. In order to maximize the 
potential of SIS micro level enablers, we suggest that there is a need to identify procedures and their relationships at the micro level between different actors. As the existing service literature does not offer much guidance in terms of how to manage human interactions to maximize the effectiveness of SIS efforts, we borrow concepts from humanistic management literature to develop our conceptual framework and typology, using three procedures to achieve the goals intended to help address the global problems that motivate the research.

\section{Theoretical Framework}

In this paper, we employ a "human dignity-centered" framework to guide social innovation efforts by service organizations at the interaction level. This framework is based on Mea and Sims' (2018) conceptualization of their human dignity business ethics perspective, which illustrates potential inputs or situations within which managers can apply underlying principles and virtues that drive actions. These principles are centered around the promotion of dignity and the common good. When applied through a cycle of processes, the framework suggests that enhanced utility and human welfare will result. We use this integrative framework of principles/values, processes, and outcomes as a way of understanding how humanistic management can be applied to SIS.

As a philosophical foundation for our human interaction approach to the humanistic formulation of SIS, we organize our circular framework around underlying foundational values that inform processes that are likely to result in our desired outcomes. Those outcomes in turn will support the foundational values, as such making the framework a coherent entity whose components are reinforcing each other. Figure 1 presents the definitional framework we use involving an organization's "configuration of principles of social responsibility, processes..., and polices, programs, and observable outcomes as they relate to the firm's societal relationships" (Wood 1991: 693). For our purposes in this present paper, this model is a "useful mechanism by which we can examine humanistic principles to guide managerial decisionmaking" (Arnaud and Wasieleski 2014: 316). Since SIS involves offering social innovations for solving global problems, a framework rooted in social responsibility is an appropriate method for organizing how humanistic management can inform SIS. We adapt this model to reflect more closely on the human dignity-centered approach for realizing the humanistic management of SIS.

\section{Underlying Foundational Values of Humanistic Organizations}

Building on Humanistic Management and Services literatures, we identify four foundational values that serve as pillars of our conceptual framework and our proposed typology. Below we discuss respect, trust, fairness and inclusion as central values that serve as the drivers for the processes of our framework.

\section{Respect}

Humanistic Management and Organizational justice fields of research point to respect as critical to both the functioning of collectives and the well-being of individuals (Huo and 


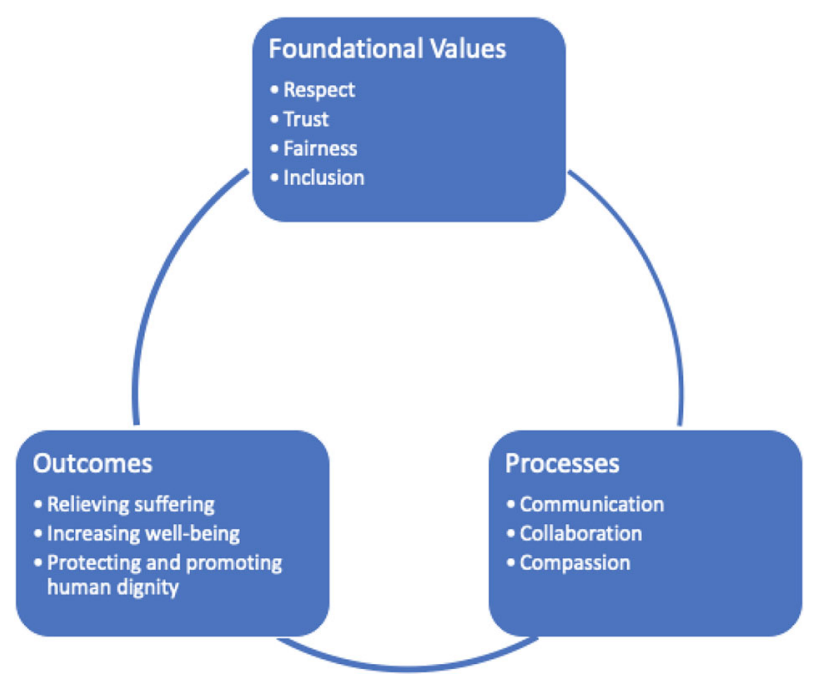

Fig. 1 The Humanistic Management Framework for SIS

Binning 2008). Empirical work examining the impact of the experience of receiving respect has demonstrated that such experiences are associated with the fulfillment of the need of belonging, which is a universal social need (De Cremer and Tyler 2005).

De Cremer and Mulder (2007: 441) proposed that "respect is considered not as something that people simply intuit by themselves, but rather as a judgment that emerges from the treatment they receive from others." Therefore, it is critical to acknowledge the role a service provider and a service receiver play in cultivating respect in service encounters.

Prior research uncovers two types of respect: Generalized or particularized respect. Generalized respect is defined as the worth accorded by one of more others which "is owed to everyone [in a social category] simply as a function of their being persons. It is not conditional on a person's status or achievements. It cannot be acquired and cannot be lost" (Lalljee et al. 2007: 452). This form of respect is inspired by Kant's view of humanity and his call to, "Act so that you use humanity... always at the same time as end and never merely as means" (2002/1785: 46-47). In contrast, particularized respect represents "the worth accorded by one or more others, which is based upon the target's attributes, behaviors, and achievements" (Rogers and Ashforth 2017: 7; Rogers et al. 2017). This type of respect is earned by the receiver on the basis of his or her valued attributes, behaviors, and achievements, fostering the sense that the individual is valued.

\section{Trust}

Trust is considered as central to successful interactions (Morgan and Hunt 1994). A large stream of literature emphasizes the role of trust as an important determinant of building longterm relationships (e.g. Doney and Cannon 1997) and of relationship quality (Moorman et al. 1992). Satisfaction with the exchange relationship or with the salesperson is positively related to trust (Anderson and Narus 1990), and cooperation between parties is positively influenced by trust (Morgan and Hunt 1994). While it is essential for exchanges in general, trust also plays 
an important role in the services context (Coulter and Coulter 2003), and in maintaining relationships with service providers as well (e.g. Kabadayi 2016).

Trust can be defined as one party's belief that another party will perform actions that will result in positive outcomes for the party, and furthermore, will not take unexpected actions that would result in negative outcomes (Anderson and Narus 1990). It also refers to the willingness of a party to be vulnerable to the actions of another party based on the expectation that the other will perform a particular action important to the trustor, irrespective of the ability to monitor or control that other party (Mayer et al. 1995). In a service setting, it can be an individual's confidence that the service organization will perform expectedly and provide services that would result in positive outcomes for the individual.

Although some researchers have treated trust as a unitary concept, most agree that trust is multidimensional with two interrelated components: trusting behavior, a willingness to depend on or make oneself vulnerable to the partner and trusting beliefs, comprising perceptions of the trustworthiness of the partner (McKnight et al. 2002). While trust in general is an important construct, different scholars emphasizes the importance of trusting beliefs in the formation of trust as they are the trusting party's perception that the trustee processes characteristics that would benefit the trusting party (McKnight and Chervany 2001). They represent a "sentiment or expectation about an exchange partner's trustworthiness" (Moorman et al. 1992). In other words, they collectively reflect the perceptions of the trustworthiness of the object of trust (McKnight et al. 2002). Mayer et al. (1995: 717) note that these factors are "not trust per se," but they help build the foundation for the development of trust.

For humanistic management, the individual is viewed as a relational being who is naturally driven to "bond" (Lawrence and Nohria 2002) and engage in social interactions. These social relationships are valued for the long-term and are not seen as one-time iterations (Pirson and Lawrence 2010). For long-term relationships to be nurtured and preserved, trust is needed between interacting parties. Cooperation between individuals is viewed as an outcome of trust (Cheng and Macaulay 2014). Thus, trust underlies the social interactions that are so critical to a humanistic management frame and service encounters.

\section{Fairness}

Another of the core values in humanistic management relates to the fair treatment of individuals in terms of upholding their dignity. Interpersonal fairness involves treating people with respect and dignity (Bies and Moag 1986). Husted (1998) made the initial connection between stakeholder fairness perceptions and the relevance for stakeholder management, noting that the former had not been previously linked to stakeholder behavior. Husted's research suggested that the creation of a mechanism based on justice theory (Rawls 1971) would allow managers to better understand the way stakeholders perceive the fairness of a firm and its "stakeholder management structures." This led to a call for organizational justice to be applied in order to analyze the relationship between firms and their stakeholders (see Hosmer and Kiewitz 2005), which has particular relevance to humanistic management.

Fairness should be based on talent, ability, experience, or effort — aspects which are thought to be relevant in distinguishing among individuals (Rawls 1971). Thus, legal requirements are not sufficient to guarantee a humanistic (fair) relationship in a service organization. Under the ethical perspective of justice, the moral act is that which affords fairness to all those affected by the decision or action. The following are dimensions of a service organization that are taken 
into consideration when employees are assessing the fairness and justice of that organization: distributive justice, procedural justice and interactional justice.

Distributive justice is concerned with the justice of outcomes and allocations (Cropanzano et al. 2007; Greenberg 1990). There are three allocation rules which need to be applied to lead to distributive justice: merit, equity, and need. As applied in service organizations, merit involves rewarding service employees based on their contributions, equality means to provide each employee with the same treatment, and need relates to provide a benefit based on one's personal requirements (Cropanzano et al. 2007; Gilliland 1993).

Procedural justice refers to the means by which outcomes are allocated in the decisionmaking process (Alder and Gilbert 2006; Cropanzano et al. 2007; Greenberg 1990). It is based on the extent to which the decision process integrates many procedural rules such as voice, accuracy, consistency, and bias suppression (Alder and Gilbert 2006; Gilliland 1993). Voice is an important factor of perceived selection fairness in a variety of contexts (Greenberg 1990). An effective two-way communication allows applicants a sense of voice regarding the selection decision (Alder and Gilbert 2006), which promotes human dignity. Accuracy is fulfilled when decisions are based on correct and concrete information (Cropanzano et al. 2007; Gilliland 1993). Consistency is also an important defining feature of procedural justice. It refers to ensuring that decisions procedures are unchanging across people and over time (Gilliland 1993). Also, the process has to be consistent across all stakeholders, meaning that they are treated the same and are subject to similar procedures (Alder and Gilbert 2006; Arvey and Renz 1992). Moreover, consistency across people refers to consistency in the content of the processes used, in evaluation (criteria), and in the interpretation of results (Arvey and Sackett 1993). Finally, interactional justice refers to how one person treats another in an organization (Cropanzano et al. 2007). There are two types of interactional justice (Colquitt et al. 2001). First, interpersonal justice relates to the respect and dignity with which one treats another. Second, informational justice means to be truthful and to provide justifications when things go badly. Interactional justice involves an interpersonal component. It is the fact that people are concerned not only with outcomes and procedures but also with the quality and the respect of the interpersonal treatment they receive in the enactment of procedures (Alder and Gilbert 2006). Fairness is obviously fulfilled when people are treated with dignity, respect and politeness.

\section{Inclusion}

Service interactions take place within broader social and economic contexts in which discrimination occurs across multiple levels and on the basis of intersectional identity characteristics, a reality that can lead to a variety of negative outcomes for marginalized communities (Seng et al. 2012). In response to the widespread unfairness within service interactions (e.g., systemic bias, customer vulnerability, marketplace discrimination, among others), Fisk et al. (2018) advance the concept of service inclusion as "an egalitarian system that provides customers with fair access to a service, fair treatment during a service, and fair opportunity to exit a service (p. 835).

Drawing upon TSR's focus on uplifting well-being through service, Anderson et al. (2013) propose four major pillars of service inclusion: enabling opportunity, offering choice, relieving suffering, and fostering happiness. To be fully inclusive, service organizations need to achieve all these four pillars in their interactions with their customers. The "enabling opportunity" 
pillar refers to "empowering people by providing access to services and the ability to receive and co-create valued services" (p. 843). The "offering choice" pillar emphasizes the right of people to make a viable choice between different offerings as well as the right to opt out of a particular service/interaction. The "relieving suffering" pillar emphasizes the importance of fulfilling basic human needs in diverse service interactions. Finally, the "fostering happiness" pillar highlight the importance of pleasure and positive interactions between actors.

As such, all four pillars provide important insights as to the "values" that should govern a human interaction/organization. Therefore, it is proposed that for a human interaction to be inclusive, actors must first and foremost recognize the likelihood that, if unchecked, service inclusion will be lacking. From there, they should empower each other, offer choice, consider the satisfaction of the basic needs, and conduct the interaction in a positive and uplifting environment. Within service encounters, or touchpoints, wherein customers interact with service providers, it is the responsibility of both parties to consider and respect the service inclusion pillars. A greater emphasis is also placed on the organization to design its processes in a manner that promotes multilevel inclusion and permits actors to realize value. Fisk et al. (2018) emphasize the fundamental role of service inclusion in advancing and improving service relationships and interaction. They argue that by acknowledging the human needs for inclusion and designing services meeting those needs, human progress becomes more plausible.

\section{Processes}

Based on the commonly used processes in the extant humanistic management literature, we identify and include three processes in our conceptual framework and our proposed typology. We discuss communication, collaboration, and compassion next.

\section{Communication}

The concept of communication can be defined in a variety of ways. In the most general terms, communication involves a sender, a channel, a message, a receiver, and some context in which the communication occurs (McQuail and Windahl 2013). In the study of communication dynamics, broadly speaking, and of communication in organizations, specifically, a "process" or "transmission" model has historically dominated scholarly thought. This perspective focuses on the effects of information exchange from a sender to a receiver, the impacts of contextual noise and feedback on the communication process, and the ability of communicative messages to be distributed for the purposes of impact and control (Craig 1999; Carey 1989). In terms of humanistic management of SIS, then, the process view of communication asks whether messages are clearly delivered at the interaction level and whether the transmission of this information has the desired effect to achieve the intended service goals.

With that said, an alternative view of communication calls attention not only to the effects of messages delivered from sender to receiver, but also to the ways that communication creates and recreates the very structures that form the foundation of organizations and interactions. This "constitutive" or "cultural" approach applies a social constructionist lens to communicative phenomena, emphasizing how meaning is co-constructed through the use of language and 
symbols by multiple communicators at multiple levels (Putnam et al. 2009). This perspective reframes communication as the ritualized maintenance of social interaction over time, a way to not only impart information but to also create and represent shared beliefs between and among communicators (Carey 1989; Rothenbuhler 1998). In terms of humanistic management of SIS, the constitutive view of communication asks how cultural understandings are developed in the communicative process, and what the social implications of those cultural understandings are for the service interaction.

While often conceptualized as opposing frameworks for the study and practice of communication, the process and constitutive approaches can be useful as complementary perspectives in conceptualizing excellent SIS. The process view calls attention to the importance of clear communication that leads to the effective transmission of information en route to achieving service goals. The constitutive approach points to the co-constructed nature of knowledge and culture, demonstrating how social innovation can be achieved when communicators reach mutual understandings through communicative interaction.

\section{Collaboration}

Optimal human interaction is not possible without collaboration with others. The humanistic perspective on organizing views leadership as "the art of building and sustaining relationships with all the relevant stakeholders..." (Pirson 2017a, b: 93). This notion is rooted in evolutionarily derived "natural" drives that govern human nature (Lawrence and Nohria 2002). One of the four main drives, the Drive to Bond, involves the innate tendency of individuals to "form social relationships and develop mutual caring commitments with other humans..." (76). A collaborative process in organizations includes building and nurturing an organizational culture that promotes interpersonal relations through integrity and trust across stakeholders (Nohria et al. 2008). Collaboration sees value in stakeholders working together. Results of co-work are additively more productive than simply combining individuals' autonomous efforts (Phillips et al. 2000). For humanistic management, organizations must be recognized as a "community of persons" because individuals are social organisms and need others to survive and flourish (Mintzberg 2009). As stated by Albert and Perouma (2017: 39), "Community entails taking care of work, colleagues, and one's place in the world."

Phenomenologically, the social psychology literature recognizes that individuals belong to social groups and often work collaboratively to achieve socially oriented goals that go beyond individual self-interest (Malena and Heinrich 2007). In the quest for new knowledge, for instance, individual persons have a greater capacity for generating possibilities when they can learn and interact with one another, sharing ideas and perspectives (To and Ko 2016). Knowledge convergence can occur when collaboration takes effect across teams with diverse backgrounds (Benavides-Espinosa and Ribeiro-Soriano 2014).

Procedurally, collaboration should take place cross-functionally where diverse individuals are able to empathize with one another and where organizational mechanisms are designed to permit consensus. The structure of the collaborating unit must have specific rules and procedures in place that specify individual roles toward a common goal. Since collaboration is a service organizational process whereby individuals can address challenges unable to be solved separately, governance mechanisms must also exist that reward team coalescence and effort and not self-interested outcomes (To and Ko 2016). Processes that promote collaboration help to develop and maintain coalitions among team members in a service organization. 
Furthermore, collaboration in the form of resource exchange and integration lies at the heart of service interaction between service employees and customers and the subsequent value cocreation (Vargo and Lusch 2008). Promoting and nurturing such collaboration between different actors in a service interaction would improve the quality of outcomes associated with that interaction.

\section{Compassion}

Compassion goes beyond a social emotion-i.e., a feeling that is aroused by real, imagined, anticipated, or recalled encounter with others (Worline and Dutton 2017) to denote the openness to the suffering of others coupled with a commitment to alleviate such suffering (Frost 2003). Prior literature describes compassion as a four-stage process that involves (1) noticing someone's suffering, (2) feeling empathy for the sufferer, (3) engaging in sense-making about the suffering, and (4) being moved to act in some way to alleviate the suffering of another person (Dutton et al. 2014). This framework situates compassion as "an interpersonal process in which both the sufferer and the focal actor play a role in how compassion unfolds over time" (Dutton et al. 2014: 278). Prior research suggests that compassion is exhibited in micro occasions of person to person interactions (Dutton et al. 2007). These micro occasions emerge from the connection between and among people representing small units of relational micro moments that are short and momentary and need not imply closeness or intimacy (Dutton and Heaphy 2003). This aspect of our framework calls attention to the cognitive and emotional openness both sides of service encounters exhibit, coupled with the actions both sides may take in attempt to alleviate distress endured by both parties.

Compassion literature sheds light on a number of emergent organizing dynamics at the heart of compassion organizing. Madden et al. (2012) propose that the emergence of organizational compassion capability is first triggered by pain triggers that becomes a new reference point for action. Under such conditions, organizational members modify their roles to include noticing, feeling empathic concern and responding to others' pain, interact with other organizational members to amplify their role, modify existing norms and coordinate compassionate responses to bring about coherent actions. According to this theory the association between pain triggers and adjusted actions is moderated by organizational members' diversity, role interdependence and the nature of social interaction between the individuals involved. Dutton et al. (2006) propose that individual compassionate responses to others can become socially coordinated through the process of compassion organizing. This process is enabled by (1) contextual enabling of attention, emotion and trust via organizational values, leader's action and organizational networks (2) agents improvising structures such as improvised routines or roles and (3) symbolic enrichment offered by the diffusion of caring stories. Together these enabling mechanisms enhance the scope, scale, speed and customization of organizational compassionate response.

\section{Outcomes}

Following our general framework of foundational values, processes, and outcomes, in this section we introduce the outcomes of TSR and Humanistic Management. 


\section{TSR Outcomes}

TSR highlights the potential of services to create uplifting changes and improvements in the well-being of consumer entities including individuals, both consumers and employees, communities, society and the entire ecosystem (Anderson et al. 2013). The feature that distinguishes TSR from other service work is the outcomes that it investigates. Instead of focusing on outcomes like profit, market share, or customer loyalty, it provides a framework for consumers' and employees' well-being on an individual and collective level, established and maintained by any type or level of interactions between different service and consumer entities (Anderson and Ostrom 2015). TSR and its outcomes are not limited to interpersonal encounters but also includes any encounter with service entities taking place during a value creation process; be that at the individual (micro), collective (meso) or at the broader ecosystem level (macro) (Anderson et al. 2013).

In general, TSR focuses on two types of well-being, hedonic and eudaimonic. Hedonic well-being refers to maintaining individual and collective happiness and defines well being in terms of pleasure attainment and pain avoidance (Ryan and Deci 2001). In the context of TSR, hedonic well being outcomes include the presence of life satisfaction and joy in employees and consumers, and the absence of negative affect such as tension, fear, and stress. Eudaimonic well-being, on the other hand, describes and emphasizes the realization of someone's potential (Ryff and Keyes 1995). It is about human flourishing that results in quality of life (Haybron and Haybron 2008), as conceptualized as the development of human capabilities and freedom (Sen 1999). Within TSR, eudaimonic well-being is applied at the individual and collective levels, and includes outcomes like access to services, literacy, better decision making, health, a decrease in health and well-being disparities, consumer involvement, harmony, power, support, and social networks (Anderson et al. 2013; Kuppelwieser and Finsterwalder 2016).

Recently, in addition to well-being, another outcome, relieving suffering, has been offered as an extension to TSR outcomes (Nasr and Fisk 2019). While improving well-being is a noble and important goal that service provision and researchers should aim to pursue, it also assumes that a certain level of well-being already exists. However, the reality is different in those contexts in which millions of people live in poverty, lacking access to most basic services needed to survive. Therefore, relieving such suffering should be included as an outcome in the TSR framework (Nasr and Fisk 2019).

\section{Humanistic Management Outcomes}

Humanistic management involves "recognizing what people are, treating them accordingly and fostering their development" (Mele 2012). The humanistic narrative starts with the realization that dignity is central to what it means to be human; suggesting that individuals need dignity in order to flourish (Pirson 2017a, b). This perspective goes beyond homo economicus assumptions about humans and portrays people as both autonomous and social. So, while humans require affiliation with others, they are also independent beings. These two aspects of the conception of individuals are viewed as interdependent and universal (Lawrence and Nohria 2002).

At the epicenter of humanistic management is the understanding and value of promoting human dignity because it has intrinsic value. Human dignity "is the idea that every human being has transcendent value that resides within his or her essence" (Mea and Sims 2018: 3). In 
a Kantian sense, individuals should be treated as ends in themselves, which implies a strong concern for the respect of their inalienable human rights. Every human being deserves to be treated with respect and should have the opportunity to enhance their own personal development (Mele 2016). Promoting dignity is necessary for achieving the common good in a society or organization (Sison et al. 2016). Without the root assumption of human dignity, the outcomes of individual well-being and flourishing cannot result.

The first humanistic management outcome that is generated out of a concern for human dignity is well-being. Based on the premise that eudaimonia is dependent on the development of dignity (Donaldson and Walsh 2015), well-being creation requires the promotion of dignity (Sen 1999). As a counter to the economic models of generating material wealth, the creation of well-being involves qualitatively measuring non-material aspects of life that lead toward life satisfaction. For instance, John Stuart Mill and Jeremy Bentham included happiness in their measurement of utility (Rosen 2012). So, while the operating logic of the economistic models is wealth creation and power driven by maximization, humanistic models have the promotion of practical wisdom and the protection of dignity through well-being as the underlying logic (Pirson 2017a).

In the psychology literature, psychological well-being refers to the felt emotion that is associated with an individual's personal growth and development, including a person's perception of his/her engagement with the self and others (Osafo Hounkpatin et al. 2015). It is normally operationalized through a combination of autonomy, self-acceptance, environmental mastery, personal growth, positive relations, and having a sense of life goals (Ryff and Keyes 1995). Thus, relationships and interaction with others are crucial in the measurement of one's personal well-being. Using a humanistic management lens, Pirson $(2017 \mathrm{a}, \mathrm{b})$ reinterprets our understanding of social welfare by viewing it as well-being creation at the organizational level.

The other outcome of the humanistic management model is flourishing. Related to wellbeing, human flourishing acknowledges that every individual's life has purpose, which is to achieve happiness. Flourishing entails understanding what end goals a person wishes to achieve and that each person has the natural right to utilize his/her abilities to their fullest potential. Based in Aristotle's teachings, flourishing involves obtaining happiness through selfactualization and autonomy to pursue moral excellence, the ultimate goal. Eudaimonia is desired as an end goal in itself and it directs all human actions. Humanistic management argues that the firm should be modeled in such a way as to allow for the self-determination of individuals to achieve their own flourishing (Pirson 2017a, b).

Based on the above summaries of outcomes, we see the TSR framework as being ontologically consistent with the humanistic management perspective. Therefore, by merging outcomes from both literatures, we suggest that service organizations that engage in social innovation efforts need to aim not only to reduce suffering and improve well-being, but also to protect and promote human dignity (Fig. 2).

\section{Proposed Typology of Human Interactions and their Relationship to the Outcomes}

We propose that reducing suffering, improving well-being and promoting human dignity can be achieved when service organizations utilize active communication, collaboration and compassion. We argue that the use of these three processes in tandem is critical to maximize 
Fig. 2 Proposed Typology

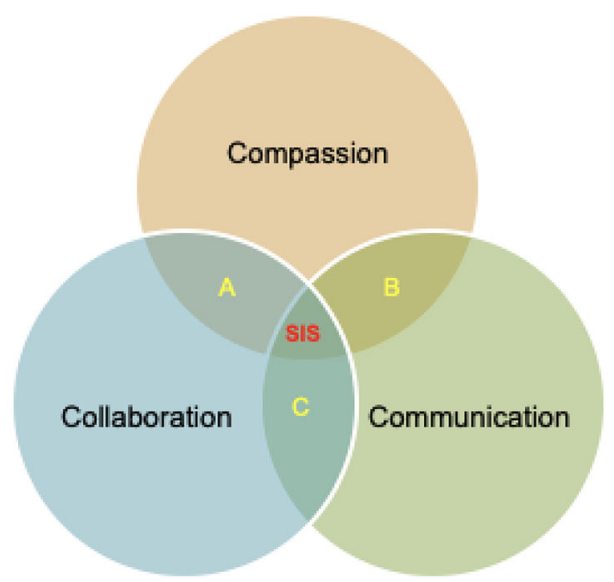

social innovation outcomes. Below we describe three typologies A, B, and C - mapping suboptimal ways by which only two of the three processes are used, and offer insights on the reasons such configurations of processes may lead to some but not all outcomes of interest. We then describe typology $\mathrm{D}$, in which service organizations are built on open communication, collaboration and compassion and describe how this configuration of processes can lead to the reduction of suffering, improvement of well-being and the promotion of dignity. We also include examples for each typology to illustrate how these organizations may or may not achieve their intended outcomes based on the processes included in each typology.

\section{Typology A: Service Organization with Compassion and Collaboration, but no Communication = Outcomes Lack Understanding}

It is possible for a service interaction that aims to help those in need to be grounded in both compassion and collaboration but also to lack ideal communication. While compassion attunes service organizations to the suffering of others and signifies a commitment to improve wellbeing, and collaboration creates a procedure to collectively identify problems and create solutions, the absence of optimal communication ultimately leads to an absence of understanding. This gap will prove a detriment to humanistic management, constraining the possibilities for social innovation by failing to reflect core values of trust, fairness, respect, and inclusion. From there, the ideal outcomes of promoting dignity, reducing suffering, and enhancing well-being are unable to be fully realized in the service context.

Taking a process or transmission view (Carey 1989; Craig 1999), ineffective communication will mean a disconnect in delivering the intended message from the service provider to the service recipient, and vice versa. In this sense, every effort can be made to offer compassion and engage in a collaborative interaction, but without optimal communication, the outcome will be hollow, leaving one or more people involved in the interaction feeling unheard or misunderstood. Without having a detailed and proper understanding of the needs of those suffering, it would be very difficult, if not impossible, for an organization to create and deliver the needed solution for consumer entities. Even with compassion and collaboration, such lack of communication in efforts may lead to suboptimal outcomes. Alternatively, the organization may think that they have offered the right solution for the problem in hand, but due to a lack of understanding of the problem or context, they may miss the point with their efforts. 
Furthermore, without good two-way communication, service providers might believe that their message is being delivered as planned, through what they understand to be a humane interaction, but the consumer entities that receive that service may see the interaction as lacking respect and not being fully inclusive. Further, without proper communicative feedback, it will be difficult to create socially innovative services that advance the core value of justice.

Taking a constitutive view (Putnam et al. 2009), gaps in communication can also lead to a deficiency of understanding in terms of the cultural connections between service organization and consumer entities. Even with compassion and collaboration, the service organization can be still seen as ignoring or misunderstanding ritual aspects of the interaction, demonstrating disrespect for the cultural knowledge of those consumer entities and falling short of inclusivity, even if unintentionally. Here, the shared, meaning-making function of communication is overlooked, a rift that would prevent service providers from fully conceptualizing the broader socio-cultural implications of their interactions with individuals, communities or society at large. Without a communicative commitment to the co-construction of knowledge, the prospects for achieving outcomes with those social innovation efforts will be limited.

As an example, a lack of communication could constrain the ability of a company that is engaged in global humanitarian efforts to provide treatment and prevention services for infectious diseases like HIV. Even though compassion may be present as a motivating force for action, and the company may collaborate with local constituents as project partners, a lack of open, two-way communication would prevent a proper understanding of the causes of the problem at hand. Indeed, communication constraints would prevent a full understanding of the social practices that lead to the transmission of the virus among local residents, as could a lack of understanding regarding cultural norms and taboos related to sex and sexuality. Offered solutions would likely be short-lived and would be unable to sustainably relieve suffering.

\section{Typology B: Service Organization with Compassion and Communication, but no Collaboration = Outcomes Lack Participation}

Service interactions can be grounded in both compassion and communication but lack collaboration among the members of a service encounter. Under such circumstances, we predict a lack of participation in the human interactions. While service providers may be cognitively aware and emotionally charged about the well-being of others and are able to interact at a communication level to promote understanding among the collective, it could be that no collaborative effort to work together is leveraged to achieve the TSR and humanistic management outcomes. Thus, a problem with lack of participation and enactment results as service providers do not collaborate with others, regardless of an underlying compassion and active communication. Humans' natural capacities for compassion and empathy should positively influence cooperation and altruism among individuals (De Waal 2013). In a sense, this participation failure is, in part, a symptom of the Drive to Bond (Lawrence and Nohria 2002) being suppressed. A community of persons means taking care of others (Albert and Perouma 2017). As such, in this instance we see a lack of community not only between the service organizations and the beneficiaries of the innovation but also between that organization and other actors like companies, government agencies, and NGOs that try to help the same group of individuals and communities. Not collaborating with those other actors may impede the success of those social innovation efforts as those efforts may miss possible synergies that can be created through participation from others, or may replicate the same efforts causing inefficiencies. 
One possible reason for this absence of community may be due to a lack of trust among service organizations and the others including the beneficiaries. Meaningful collaboration requires that parties to the interaction develop trust through dialogue (Mele and SanchezRunde 2011). Finally, lack of collaboration can create an interaction where the service provider demonstrates power over rather than power with others (Miller and Stiver 1997), which would result in a paternalistic approach toward beneficiaries. While it may still provide some well-being outcomes for individuals and communities, such one-way approach may not be aligned with the core values like respect and inclusion. Also, due to this lack of participation those individuals and communities for whom those efforts are intended may not feel ownership for those solutions or they do not internalize them, and thus the effectiveness of social innovation efforts may be limited. Finally, when lacking participation, that kind of one-way approach that aims to help those consumer entities may hurt their dignity or unintentionally deprive them of their dignity as they find themselves as passive recipients of those innovative solutions that aim to help without any participation in them.

An example of a service organization in this typology could be a service provider that tries to design and deliver offerings to alleviate suffering and improve well-being of refugees in a dignified way. Even though such an organization would have the compassion to help those refugees and communicate with them to better understand their needs, unless they also include collaboration as a key process in their efforts they may not achieve their intended goals. For example, failing to collaborate with the local population that hosts those refugees may result in creating unintentional hostility from locals toward the same refugees, as those locals may consider such efforts unfair or just depletion of resources that could be used otherwise to support them. Similarly, lack of collaboration with other government agencies and organizations that also try to help those refugees may result in replicated efforts and wasting some very valuable resources that could be used in a more effective and efficient way with better planning and participation from those organizations.

\section{Typology C: Service Organization with Collaboration and Communication, but no Compassion = Outcomes Lack Emotional Connection}

Service interactions can be grounded in both collaboration and communication but lack compassion. While collaboration invites service providers and service receivers to coparticipate in problem identification and/or solutions creation, and communication enables mutual cognitive understanding of service providers and service receivers, lack of compassion reflects low empathic concern (i.e. other-oriented feelings that are most often congruent with the perceived welfare of the other person" (Batson 1994: 606) and ultimately leads to poor emotional connection (Brown 2012) between service providers and service receivers. We argue that this emotional detachment will impede humanistic management and constrain the possibility for social innovation and as it undermines one's likelihood to act to protect and promote human dignity and as well as reduce one's motivation to act to relieve others' suffering or uplift others' well-being.

Lack of empathic concern and felt emotional connection of service providers toward service receivers can shape social innovation service encounters. Felt connection and empathic concern for a service receiver can foster a more complete and accurate understanding of the service receiver (Hakansson and Montgomery 2003). According to the empathy gap theory (Loewenstein 1996), when individuals fail to accurately assess the emotional burden of a 
distress, they underestimate the severity and difficulty of a distressed endured by others. Therefore, emotionally detached service providers, who lack empathetic concern for service receivers, may underestimate the severity, difficulty of a distress endured by others and thus demonstrate biased understanding of the urgency of such needs. We propose that service encounters built on such an approach can threaten the dignity, generalized respect, and interactional justice experienced by a service receiver. Moreover, conceptual and empirical evidence indicates that empathic concern evokes the motivation to help others (Batson 1994; Lazarus 1991). In particular, emotionally relating to another's distress increases the inclination to advance the other individual's welfare (Batson and Shaw 1991; Dutton et al. 2014). Thus, service providers who feel emotionally detached and demonstrate low empathic concern toward individuals and communities are likely to demonstrate lower motivation to relieve others' suffering and uplift others' well being- both of which are key social innovation outcomes.

For example, a service organization in this typology would be one that tries to provide healthcare services to those in rural regions in the developing world experiencing poverty. It may have processes in place that help communicate with various entities involved to deepen their understanding of the problem, and build collaborations with local authorities and people in need, but may fall short on compassion. Extensive evidence suggests that in the process of offering empathic support, doctors, nurses and first responders can personally experience the pain of their patients and families, creating secondary trauma that can lead to service providers' compassion fatigue (i.e. physical, emotional and spiritual depletion associated with caring for others in significant physical and emotional distress, Figley 1995). Under such circumstances, service providers may avoid working with certain patients, demonstrate increased absenteeism and exhibit lack of joyfulness for service work- that together can hamper the effectiveness of the health service effectiveness and unintentionally trigger service providers' suffering and deplete their well-being. Furthermore, when service organizations approach a social innovation service project with limited compassion, they may do so not because such social innovation is an important component of their company mission and values, but because engaging in such activity can be beneficial for publicity and goodwill.

\section{Typology SIS: Service Organization with Compassion, Communication and Collaboration $=$ Maximized Outcomes for Social Innovation Efforts}

We argue that service interactions involving a combination of compassion, communication, and collaboration are most likely to result in the maximization of outcomes for the SIS efforts in our framework. This means that a service interaction that includes all three elements will lead to improved well-being - eudaimonic and hedonic - as well as the relief of human suffering and the promotion of human dignity. Thus, the desired TSR and humanistic management outcomes require the existence of all three processes.

For well-being to be improved and human suffering to be mitigated requires an empathetic understanding of other people's' suffering, entailing a full cognitive-affective process. Compassion capability is viewed as a prerequisite for compassion organizing (Pirson 2018), which is the collective response to a specific incidence of suffering involving the coordination of individuals' compassion (Dutton et al. 2006). At the human interaction level, this assumes collaboration among individuals aware of and concerned about the suffering. The idea of compassion organizing also necessitates proper communication among these empathetic persons. Thus, the dialogue must also involve a communication of trust among interacting 
parties to promote collaboration. Trust plays a role in facilitating the formation of long-term networks of people and groups (Nielsen 2004; Ring 1996). Thus, to organize a compassionate response to human suffering and the promotion of well-being, the members of the collective must first feel empathic concern toward others, but then must communicate with each other to achieve sensemaking of the incident. Action toward the relief of the suffering then needs collaboration among the members of the collective.

Compassion capability can be viewed as a norm possibly derived from evolution to enhance a human's chances of survival and flourishing (Pirson and Lawrence 2010). The idea here is that humans are social beings that need to bond with each other to realize their full potential. Well-being and reduction of suffering are byproducts of human flourishing. Compassion for others involves treating humans as ends in themselves which is a key component of dignity (Mea and Sims 2018). With the protection of dignity as the baseline of humanistic management thought (Pirson 2017a, b), eudaimonic well-being is possible. "Human dignity potentially serves as common ground in our efforts to identify and secure local and global common interests in an increasingly interconnected world" (Mattson and Clark 2011: 304). It serves a crucial role as a fundamental value in the process of making relationship more humanistic (Pless et al. 2017).

We posit that these outcomes interact. However, Pirson (2018) argues that compassion may not be hardwired into human capabilities; it is something that needs to be nurtured and learned. Thus, to achieve these collective outcomes, compassion must be coupled with communication and collaboration in order to learn from each other. Human interaction is critical.

An example of a service organization that embodies and enacts compassion, communication, and collaboration to maximize social innovation outcome can be found in Greyston, a hybrid organization founded in 1982 as a "place of opportunity for everyone... based on Buddhist tenets of non-judgment and loving action” (Laasch et al. 2019). The nonprofit entity oversees a for profit bakery and includes programming for workplace educational development, all operating with "the mission to create thriving communities through the practice and promotion of Open Hiring" (Laasch et al. 2019), a philosophy and practice informed by the founders Zen-Buddhist principles articulated above. Greyston is committed to act to alleviate the suffering of individuals who otherwise may not be suitable for employment, protect and promote dignity via their commitment to Open hiring philosophy and practice- which includes providing open access to work opportunities and/or expansion of hard and soft-skills to enable employment at Greyston (or elsewhere in the community), and uplift individual well-being and by extension community well-being. To achieve these goals at the bakery, Greyston employs an Open Hiring Model. Through this process, Greyston opens its doors to those who seek employment, refrain from background checks, and selection processes and focuses on open communication with all parties involved to clearly express expectations, understand employees skills, needs, interests and aspirations to enable employees' to either retain their opportunity at Greyston or seek other employment, a practice rooted in Greyston's PathMaking philosophy which respects an individual's path and allows for self-determination on individuals to achieve their own flourishing. Further, the PathMaking philosophy comes to life when Greyston employees work with participants/clients, rather than imposing assistance/services on anyone, ensuring participants exercise choice at every interaction with Greyston. This approach further ensures Greyston enables active participation from service participants, to ensure the participants feel ownership over their own flourishing and well-being.

Greyston acknowledges the unique (or systematically-imposed) life related hurdles that can impede one's ability to obtain or retain a job and is thus committed to compassionately assist 
employees to deal with and overcome such difficulties. Beyond the willingness to support, genuine care and mentorship supervisors, and HR and workplace development personnel, Greyston compassionately supports Bakery employees in need by partnering with local agencies to have a social case worker placed at their facility to assist employees overcome personal challenges and/or navigate complex social service systems to meet their needs. Greyston works collaboratively with agencies and employers in the greater New York area to create generative pathways for Greyston employees and workplace development program students, enabling such individuals' placements outside of Greyston boundaries. Such collaborations open new opportunities for individuals and enhances person-job fit in placement which is central for long term employment. Importantly, employing the "unemployable" and working toward employment pathmaking does not only benefit individuals employed and serviced by Greyston but also creates ripple effects for family members of such individuals and their community.

\section{Discussion and Implications}

Human beings all over the world are undoubtedly facing much bigger and more complicated problems than ever before. In fact, the scope and urgency of the solutions needed to deal with those problems is evidenced by the direct involvement of the United Nations with their declaration of the Sustainable Development Goals. While there have been efforts to solve global problems from various government agencies and NGOs all over the world, given the centrality of the private sector to modern society, it is clear that there is a need for productive involvement of for-profit companies in the search for solutions. Within this context, service organizations have a great potential to contribute to those efforts. As discussed earlier, with their recent paper, Aksoy and colleagues (Aksoy et al. 2019) coined a new concept, Social Innovation in Service (SIS), to highlight the role service organizations can play in creating and offering market-based solutions to societal problems. By bridging the services and humanistic management literatures, this paper developed a conceptual framework and proposed a typology where service organizations can use three different procedures (i.e. collaboration, communication and compassion) to maximize the outcomes of their social innovation efforts. More specifically, the proposed typology suggests that those social innovation outcomes can be maximized only when a service organization has all three procedures operating together. In cases in which collaboration, communication and compassion are all present, the interaction between those procedures enables the social innovation efforts of service organizations to be maximized. However, in other cases, when one of those procedures is absent, such efforts would either lack agency, understanding or emotional connection, which would hamper the full realization of their efforts and constrain the potential of subsequent outcomes.

In addition, this paper also contributes to the humanistic management literature by adopting service literature's focus on the human interaction as a level of analysis, and thus filling the gap in terms of the missing human interaction level analysis in its analyses and procedures. While the humanistic management framework emphasizes well-being and human dignity as two critical organizational outcomes, our framework suggests that including human interactions in the design and management of those organizations would help them achieve their intended outcomes.

In a service setting various types of human interactions may take place: employee to customer (EC), employee to employee (EE) and customer to customer (CC). The importance 
of incorporating communication, collaboration, and compassion across those various types of human interactions is essential for true SIS success. Even though interactions under the control of the organization could seem to be easier to infuse with communication, collaboration and compassion through proper management and leadership (i.e. EC and EE), organizations can lead by example and hope this would encourage customers to adopt similar values through the spillover effect. As such adopting the framework by all the actors will have a direct and indirect positive impact on everyone.

Finally, our proposed framework is the first attempt at bridging two different disciplines, humanistic management and service research, that share the same aspirations to address global problems. The overlap between the outcomes of humanistic management framework and TSR within service research offers a promising avenue for further collaborations between two disciplines. In the future, both humanistic management and service scholars may explore further opportunities for collaboration to address issues that the humanity faces.

Our framework and proposed typologies offer some relevant managerial implications for service organizations that are engaged in social innovation efforts to provide relief for some of the global problems that we collectively face. First of all, as our typology suggests, the outcomes of social innovation efforts can be maximized when service organizations use all three processes simultaneously. While different combinations of those processes are used in different organizations, it is critical to remember that the simultaneous existence of all three is what leads to maximized outcomes. Therefore, an internal audit to make sure that a service organization has indeed all those processes in place even before initiating social innovation efforts would be an important step to achieve intended goals of relieving suffering, improving well-being, and promoting dignity. Our ideal typology also suggests that in cases where one process is missing, service organizations will likely fall into one of the other three sub-optimal typologies and exhibit lack of understanding, participation or emotional connection. Thus it would be imperative for service organizations seeking to move towards the ideal typology to create enabling conditions that can facilitate the promotion of the missing processes that impede such organizations from maximizing SIS outcomes.

Second, service organization that seek to maximize SIS outcomes may consider the HR selection implications for the framework we propose. More specifically, we propose that personality characteristics such as emotional intelligence (i.e. the ability of an individual to perceive accurately, appraise, and express emotion; the ability to access and generate feelings when they facilitate thought; the ability to understand emotion and emotional knowledge; and the ability to regulate emotions to promote emotional and intellectual growth (Mayer and Salovey 1997) may be central to enable the enactment of the ideal typology we describe above. Individuals who can identify and manage their own emotions and who display self-control, can serve as role models for their partners, thereby earning other's trust and respect (Modassir and Singh 2008). Moreover, individuals who are able to manage emotions in relationships can better understand others' needs, cultivate more generative collaborations and are more likely to craft solutions that better fit the needs of consumer entities. Therefore, selecting and assigning those who exhibit high emotional intelligence to social innovation roles can enable organizational efforts toward maximizing SIS outcomes.

Third, social innovation efforts by service organizations and the requirement of using all three processes simultaneously may be a demanding task and may challenge those organizations' ability to persist in such work over time. For this end, training that can help service providers to engage in self-care could be critical. Self compassion programs can be especially valuable to ensure that service organizations can sustain themselves over time, and thus 
contribute to providing solutions of those global problems. Such programs are designed to enhance service entities self-kindness, enhance service entities recognition of common humanity and practice mindfulness in a non judgmental, receptive mind state (Neff 2003, 2011).

Finally, in general, the changes we propose in this paper are indeed likely to be part of a larger culture change within organizations. We suggest that a humanistic management approach to service encounters will lead to the relief of suffering, increased well-being, through the promotion of dignity. However, it should be acknowledged that these stated outcomes have implications on the organization as well. Relieving human suffering and striving for employee well-being has positive effects on the organization and can ultimately lead to further business opportunities that increase competitive advantage. For instance, studies in human resource management have demonstrated that organizations that promote well-being are more likely to increase employee job satisfaction (Felstead et al. 2015; Rothausen and Henderson 2019). As a consequence of this relationship, firms find that increasing job satisfaction for employees results in a greater overall competitive advantage (Duserick and Huang 2013). Thus, the benefits of using our approach have positive trickle-down effects for the organization in terms of business opportunities.

\section{Potential Challenges and Obstacles}

While this paper offers a theoretical framework with three key processes to help service organizations improve the outcomes of their social innovation efforts, it is inevitable that they may face some challenges and obstacles in implementing this framework. Since such potential obstacles could impede organizations' social innovation efforts, their identification would be critical in terms of maximizing the success of these efforts. This task is especially important in social innovation in services as there are many stakeholders involved and in many cases those stakeholders can be in different countries across the world. Therefore, a detailed audit of potential obstacles should take place prior to the implementation of our proposed framework. While it is not an exhaustive list, we provide a few exemplary obstacles that organizations need to consider in their social innovation efforts to achieve their intended outcomes.

At the macro level, factors such as culture and technology could potentially impede the successful implementation of the proposed strategies within the framework of social innovation efforts by service organizations.

First, since in many cases social innovation involves designing solutions for problems in different countries and to different groups with varying cultures and social norms, these need to be fully understood to avoid potential negative or unintentional consequences. For example, local customs or religious practices may make it inappropriate to have collaboration or communication between men and women. In that case, even with the best intentions, interactions between service providers and locals of different genders may not create the intended outcomes because of a lack of cultural understanding. Similarly, questions about some topics or issues may backfire if those are considered as taboo in a specific culture. Related, cultures characterized by high power distance may constrain open, two-way communication between parties who hold various degrees of power within and outside the organization. Therefore, a detailed understanding of cultural sensitivities is essential when designing social innovation efforts. Future research should consider this aspect of the implementation of our framework across cultures.

Second, technology, especially the use of frontline service technology (De Keyser et al. 2019), could present some challenges. For services offered through different technologies, not 
only communication between organizations and recipients can be hindered, but the mediation of interaction through technology may also make it harder to have compassion present in those relationships. Furthermore, absence of technology in different contexts, or lack of its acceptance by locals in different parts of the world could, create further challenges in terms of achieving intended outcomes. Therefore, given the potential obstacles that can be created by technology and technological innovations in their social innovation efforts, organizations may want to have a detailed understanding of the affordances and constraints offered by technologies within the context of their implementation plans.

At the organizational level, two related factors might create challenges and therefore hinder the success of the proposed strategies: organizational culture and leadership style. Since the three processes included in our theoretical framework may require organization-wide efforts and resources, overall organizational culture would be an important factor to consider when implementing social innovation projects. Having the commitment and involvement not only from top management but also from middle-management levels would help with setting the tone for the rest of the organization and employees. Furthermore, the processes as described above may require more inclusive, less hierarchical management and open communication structure within organizations. Additionally, the need to be flexible and agile to provide customized solutions for different problems in different contexts would require a certain type of leadership style. Thus, performing an internal audit of the organizational culture and leadership and communication styles as part of the social innovation planning process would be useful, especially in cases where a culture and mindset change is needed. In such cases, the use of three processes, communication-collaboration-compassion, to create the necessary cultural shift within the organization would help with the success of subsequent social innovation efforts.

Finally, at the individual and human interaction level, different factors such as personal beliefs and biases, explicit or hidden, held by both employees and customers, could present challenges for the successful implementation of service organizations' plans to provide solutions to those in need. For example, regardless of the organization's overall vision, employees' lack of believing in and empathy for causes that the organization aims to help with could interfere with the successful implementation of their efforts. Alternatively, employees, while committed to the overall social innovation idea, may have different priorities when it comes to helping other people. For various personal reasons they may believe that a specific cause or problem is more important than the one that the organization has chosen for its social innovation efforts and this gap may affect their individual performance and thus the overall success of such organizational efforts.

To sum up, while adopting the framework as described above could help service organizations achieve and maximize their social innovation endeavors, they need to be aware of different obstacles that may impede their success. The scope and complexity of social innovation projects in terms of different stakeholders, cultures, and geographic locations involved require a clear understanding of such potential obstacles prior to the planning and implementation phases so those organizations can overcome them and achieve their intended outcomes.

\section{Future Research}

Future studies may want to empirically test the conceptual framework proposed in this paper in different ways. First of all, the existence of proposed typologies can be empirically validated 
by using data from service organizations and conducting a cluster analysis of those organizations based on the three processes as defined above. Additionally, the levels of social innovation outcomes of service organizations can be compared across these typologies to confirm that those outcomes are maximized when all three processes exist simultaneously, and they are suboptimal when one of the processes is absent. Furthermore, conducting a profile deviation analysis, future studies may also explore the ideal levels in combination of the three processes to maximize intended outcomes, as not only presence but also an ideal level of those processes may play a role in achieving the intended outcomes. Another research stream could be to investigate if different types or contexts of global problems may require different typologies of those processes to maximize outcomes. For example, service organizations' efforts that aim to increase access to healthcare or to provide required services to refugees may require combinations of different levels of those processes, and those levels can be identified using empirical data to provide insights for service organizations. Finally, while the proposed framework includes processes at the human interaction level, future studies may want to expand this framework by including other organizational factors such as management or leadership style or organizational goals within service organizations.

\section{References}

Akaka, Melissa Archpru, Stephen L. Vargo, and Heiko Wieland. 2017. Extending the context of innovation: the co-creation and institutionalization of technology and markets. In Innovating in Practice (43-57). Springer, Cham.

Aksoy, Lerzan, Linda Alkire, Sunmee Choi, Peter Kim, and Lian Zhang. 2019. Social Innovation in Service: A Conceptual Framework and Research Agenda. Journal of Service Management.

Albert, M.N., and J.P. Perouma. 2017. The dialogue: An essential component to consider "organization as a community of persons". Humanistic Management Journal 2: 37-55.

Alder, S.G., and J. Gilbert. 2006. Achieving ethics and fairness in hiring: Going beyond the law. Journal of Business Ethics 68 (4): 449-464.

Anderson, J.C., and J.A. Narus. 1990. A Model of Distributor Firm and Manufacturer Firm Working Partnerships. Journal of Marketing 54 (January): 42-58.

Anderson, Laurie, and Amy Ostrom. 2015. Transformative service research: advancing our knowledge about service and well-being. Journal of Service Research 18 (3): 243-249.

Anderson, Laurel, Amy L. Ostrom, Canan Corus, Raymod P. Fisk, Andrew S. Gallan, Mario Giraldo, Martin Mende, Mark Mulder, Steven W. Rayburn, Mark S. Rosenbaum, Kunio Shirahada, and Jerome D. Williams. 2013. Transformative service research: an agenda for the future. Journal of Business Research 66 (8): 1203-1210.

Arnaud, S., and D.M. Wasieleski. 2014. Corporate humanistic responsibility: Social performance through managerial discretion of the HRM. Journal of Business Ethics 120: 313-334.

Arvey, D., and G.L. Renz. 1992. Fairness in the selection of employees. Journal of Business Ethics 11 (5): 331-340.

Arvey, R.D., and P.R. Sackett 1993. Fairness in selection: Current developments and perspectives. In N. Schmitt, \& W. Borman (Eds.), Personnel Selection San Francisco, CA: Jossey-Bass.

Ashoka. 2019. https://www.ashoka.org. Accessed 20 Feb 2019.

Bailey, J., J. Dwayne, D. Gremler, and M.A. McCollough. 2001. Service encounter emotional value. Services Marketing Quarterly 23 (1): 1-24.

Batson, C.D. 1994. Why Act for the Public Good? Four Answers. Personality and Social Psychology Bulletin 20 (5): 603-610.

Batson, C.D., and L.L. Shaw. 1991. 'Encouraging Words Concerning the Evidence of Altruism. Psychological Inquiry 2 (2): 159-168.

Benavides-Espinosa, M., and D. Ribeiro-Soriano. 2014. Cooperative learning in creating and managing joint ventures. Journal of Business Research 67 (4): 648-655.

Bies, R.J., and J.S. Moag. 1986. Interactional justice: Communication criteria for justice. In B. Sheppard (Ed.), Research on negotiation in organizations pp. 35-55.

Brodie, Roderick J., Linda D. Hollebeek, Biljana Jurić, and Ana Ilić. 2011. Customer engagement: conceptual domain, fundamental propositions, and implications for research. Journal of Service Research 14 (3): $252-272$. 
Brown, B. 2012. Daring Greatly: How the Courage to Be Vulnerable Transforms the Way We Live, Love, Parent, and Lead. New York: Avery.

Carey, J. 1989. Communication as culture: Essays on media and society. Winchester: Unwin Hyman.

Cheng, Xusen, and Linda Macaulay. 2014. Exploring individual trust factors in computer mediated group collaboration. Group Decision Negotiations 23: 533-560.

Colquitt, J.A., D.E. Conlon, M. Wesson, C.O. Porter, and K. Yee Ng. 2001. Justice at the millennium: A metaanalytic review of 25 years of organizational justice research. Journal of Applied Psychology 86 (3): 425-445.

Coulter, K.S., and R.A. Coulter. 2003. The effects of industry knowledge on the development of trust in service relationships. International Journal of Research in Marketing 20 (1): 31-43.

Craig, R.T. 1999. Communication theory as a field. Communication Theory 9 (2): 119-161.

Cropanzano, R., Bowen, D.E., and S.W. Gilliland. 2007. The Management of Organizational Justice. Academy of Management Executive 21 (4): $24-48$

De Cremer, D., and L.B. Mulder. 2007. A passion for respect: On understanding the role of human needs and morality. Gruppendynamik und Organisationsberatung 38: 439-449.

De Cremer, D., and T.R. Tyler. 2005. Am I respected or not?: Inclusion and reputation as issues in group membership. Social Justice Research 18: 121-153.

De Keyser, A., S. Köcher, L. Alkire, C. Verbeeck, and J. Kandampully. 2019. Frontline Service Technology infusion: conceptual archetypes and future research directions. Journal of Service Management 30 (1): 156-183.

De Waal, F.B. 2013. The bonobo and the atheist: In search of humanism among the primates. New York: Wiley.

Dolen, Van, Ko De Ruyter Willemijn, and Jos Lemmink. 2004. An empirical assessment of the influence of customer emotions and contact employee performance on encounter and relationship satisfaction. Journal of Business Research 57 (4): 437-444.

Donaldson, Thomas, and James Walsh. 2015. Toward a theory of business. Research in Organizational Behavior 35: $181-207$.

Doney, P.M., and A.P. Cannon. 1997. An examination of the nature of trust in buyer-seller relationships. Journal of Marketing 61: 35-51.

Duserick, F., and W. Huang. 2013. Job Satisfaction as a Competitive Advantage: A Longitudinal Study. Competition Forum 11 (2): 69-81.

Dutton, J.E., and E.D. Heaphy. 2003. The power of high-quality connections. In Positive Organizational Scholarship: Foundations of a New Discipline, ed. K.S. Cameron, J.E. Dutton, and R.E. Quinn, 263-278. San Francisco: Berrett-Koehler.

Dutton, J.E., M.C. Worline, P.J. Frost, and J.M. Lilius. 2006. Explaining compassion organizing. Administrative Science Quarterly 51 (1): 59-96.

Dutton, J.E., J.M. Lilius, and J.M. Kanov. 2007. The transformative potential of compassion at work. In Handbook of Transformative Cooperation: New Designs and Dynamics, ed. S.K. Piderit, R.E. Fry, and D.L. Cooperrider, 107-126. Stanford: Stanford University Press.

Dutton, J.E., K.M. Workman, and A.E. Hardin. 2014. Compassion at work. Annual Review of Organizational Psychology and Organizational Behavior 1: 277-304.

Felstead, A., D. Gallie, F. Green, and H. Inanc. 2015. Fits, Misfits, and Interactions: Learning at Work, Job Satisfaction and Job-Related Well-Being. Human Resource Management Journal 25 (3): 294-309.

Figley, C.R. 1995. Compassion fatigue: Coping with secondary traumatic stress disorder in those who treat the traumatized. New York: Brunner-Mazel.

Fisk, R.P., A. Dean, L. Alkire (née Nasr), A. Joubert, J. Previte, N. Robertson, and M.S. Rosenbaum. 2018. Design for service inclusion: creating inclusive service systems by 2050. Journal of Service Management 29 (5): 834-858.

Frost, P.J. 2003. Toxic Emotions at Work: How Compassionate Managers Handle Pain and Conflict. Boston: Harvard Business School Press.

Gabbott, Mark, and Gillian Hogg. 2001. The role of non-verbal communication in service encounters: A conceptual framework. Journal of Marketing Management 17 (1-2): 5-26.

Gentile, Chiara, Nicola Spiller, and Giuliano Noci. 2007. How to sustain the customer experience: an overview of experience components that co-create value with the customer. European Management Journal 25 (5): 395-410.

Gilliland, S.W. 1993. The Perceived Fairness of Selection Systems: An Organizational Justice Perspective. Academy of Management Review. 18 (4): 694-734.

Grandey, A.A. 2000. Emotional regulation in the workplace: A new way to conceptualize emotional labor. Journal of Occupational Health Psychology 5 (1): 95.

Greenberg, J. 1990. Organizational justice: Yesterday, today and tomorrow. Journal of Management 16: 399-415.

Grönroos, C. 1998. Marketing services: The case of a missing product. Journal of Business and Industrial Marketing. 13 (5): 322-338

Gustafsson, A., C. Högström, Z. Radnor, M. Friman, K. Heinonen, E. Jaakkola, and C. Mele. 2016. Developing service research-paving the way to transdisciplinary research. Journal of Service Management 27: 9-20. 
Gutek, B.A., M. Groth, and C. Bennett. 2002. Achieving service success through relationships and enhanced encounters. Academy of Management Perspectives 16 (4): 132-144.

Hakansson, Jakob, and Henry Montgomery. 2003. Empathy as an Interpersonal Phenomenon. Journal of Social and Personal Relationships 20 (3): 267-284.

Haybron, D. M., and Haybron, D. (2008). The pursuit of unhappiness: The elusive psychology of well-being. Oxford University Press on Demand.

Hosmer, LaRue T., and Christian Kiewitz. 2005. Organizational justice: A behavioral science concept with critical implications for business ethics and stakeholder theory. Business Ethics Quarterly 15 (1): 67-91.

Huo, Y.J., and K.R. Binning. 2008. Why the psychological experience of respect matters in group life: An integrative account. Social and Personality Psychology Compass 2: 1570-1585.

Husted, Bryan. 1998. Organizational Justice and the Management of Stakeholder Relations. Journal of Business Ethics 17 (6): 643-651.

Kabadayi, S. 2016. Customers' dissatisfaction with banking channels and their intention to leave banks: The moderating effect of trust and trusting beliefs. Journal of Financial Services Marketing 21: 194-208.

Kant, I. 2002/1785. Groundwork for the metaphysics of morals. A. W. Wood (Ed. \& Tran.). New Haven: Yale University Press.

Kuppelwieser, V.G., and J. Finsterwalder. 2016. Transformative service research and service dominant logic: Quo vadits? Journal of Retailing and Consumer Services 28: 91-98.

Laasch, O., R. Livne-Tarandach, and M. Pirson. 2019. Responsible management unfiltered: Bakers on a mission to scale open hiring. CRME Cases 1 (2).

Lalljee, M., S.M. Laham, and T. Tam. 2007. Unconditional respect for persons: A social psychological analysis. Gruppendynamik und Organisationsberatung 38: 451-464.

Lawrence, P.R., and N. Nohria. 2002. Driven: How human nature shapes our choices. San Francisco: Jossey Bass.

Lazarus, R.S. 1991. Emotion and Adaptation. New York: Oxford University Press.

Lloyd, A.E., and S.T.K. Luk. 2011. Interaction behaviors leading to comfort in the service encounter. Journal of Services Marketing. 25 (3): 176-189

Loewenstein, G. 1996. Out of control: visceral influences on behavior. Organizational behavior and Human Decision Processes 65 (3): 272-292.

Madden, L.T., D. Duchon, T.M. Madden, and D.A. Plowman. 2012. Emergent organizational capacity for compassion. Academy of Management Review 37 (4): 689-708.

Malena, C., and V.F. Heinrich. 2007. Can We Measure Civil Society? A Proposed Methodology for International Comparative Research. Development in Practice. 17 (3). 338-352.

Mattson, D.J., and S.G. Clark. 2011. Human dignity in concept and practice. Policy Sciences 44: 303-319.

Mayer, J. D., and Salovey, P. 1997. What is emotional intelligence? In P. Salovey, \& D. J. Sluyter (Eds.), Emotional development and emotional intelligence: Educational implications (pp. 3-31). New York, NY. Basic Books.

Mayer, R.C., J.H. Davis, and F.D. Schoorman. 1995. An integrative model of organizational trust. Academy of Management Review 20: 709-734.

McKnight, D.H., and N.L. Chervany. 2001. What trust means in e-commerce customer relationships: an interdisciplinary conceptual typology. International Journal of Electronic Commerce 6: 35-59.

McKnight, D.H., V. Choudhury, and C. Kacmar. 2002. Developing and validating trust measures for ecommerce: an integrative typology. Information Systems Research 13: 334-359.

McQuail, D., and Windahl, S.. 2013. Communication models for the study of mass communications. Routledge.

Mea, W.J., and Sims, R.R. 2018. Human dignity-centered business ethics: A conceptual framework for business leaders. Journal of Business Ethics https://doi.org/10.1007/s10551-018-3929-8.

Medler-Liraz, H. 2016. The role of service relationships in employees' and customers' emotional behavior, and customer-related outcomes. Journal of Services Marketing 30 (4): 437-448.

Mele, Dominec. 2012. The firm as a "community of persons": A pillar of humanistic business ethos. Journal of Business Ethics 106: 89-101.

Mele, Dominec. 2016. Understanding humanistic management. Humanistic Management Journal 1: 1-23.

Mele, Dominec, and Carlos J. Sanchez-Runde. 2011. Towards a holistic understanding of management. Journal of Management Development 30 (6): 544-547.

Miller, J., and I.P. Stiver. 1997. The healing connection: How women form relationships in therapy and in life. $1 \mathrm{st}$ ed. Boston: Beacon Press.

Mintzberg, Henry. 2009. Rebuilding companies as communities. Harvard Business Review 87: 140-143.

Modassir, A., and T. Singh. 2008. Relationship of Emotional Intelligence with transformational leadership and organizational citizenship behavior. International Journal of Leadership Studies 4 (1): 3-21.

Moorman, C., G. Zaltman, and R. Deshpande. 1992. Relationships between providers and users of market research: the dynamics of trust within and between organizations. Journal of Marketing Research 29: 314-328.

Morgan, R.M., and S.D. Hunt. 1994. The commitment-trust theory of relationship marketing. Journal of Marketing 58 (3): 20-38. 
Nasr, L., and R. Fisk. 2019. The Global Refugee Crisis How Can Transformative Service Researchers Help? The Service Industries Journal. https://doi.org/10.1080/02642069.2018.1445224.

Nasr, L., J. Burton, and T. Gruber. 2015. When good news is bad news: the negative impact of positive customer feedback on front-line employee well-being. Journal of Services Marketing 29 (6/7): 599-612.

Neff, K. 2003. Self-compassion: An alternative conceptualization of a healthy attitude toward oneself. Self and Identity 2 (2): 85-101.

Neff, K. 2011. Self compassion: The proven power of being kind to yourself. NY: Harpercollins Publishers.

Nielsen, B.B. 2004. The role of trust in collaborative relationships: A multi-dimensional approach. M@n@gement 7 (3): 239-256.

Nohria N., Groysberg B., and L.E. Lee 2008. Employee motivation: a powerful new model. Harvard Business Review. 86 (7-8): 78-84

Osafo Hounkpatin, H., A.M. Wood, C.J. Boyce, and G. Dunn. 2015. An existential-humanistic view of personality change. Social Indicators Research 121: 455-470.

Ostrom, A.L., M.J. Bitner, S.W. Brown, K.A. Burkhard, M. Goul, V. Smith-Daniels, H. Demirkan, and E. Rabinovich. 2010. Moving forward and making a difference: research priorities for the science of service. Journal of Service Research 13 (1): 4-36.

Patterson, P.G., E. Cowley, and K. Prasongsukarn. 2006. Service failure recovery: the moderating impact of individual-level cultural value orientation on perceptions of justice. International Journal of Research in Marketing 23 (3): 263-277.

Phillips, N., T.B. Lawrence, and C. Hardy. 2000. Inter-organizational collaboration and the dynamics of institutional fields. Journal of Management Studies 37 (1): 23-43.

Pirson, M. 2017a. A Humanistic Perspective for Management Theory: Protecting Dignity and Promoting WellBeing. Journal of Business Ethics: 1-19.

Pirson, M. 2017b. Humanistic management-protecting dignity and promoting well being. Cambridge: Cambridge University Press.

Pirson, M. 2018. Exploring the boundaries of compassion organizing. Humanistic Management Journal 2 (2): 151-169.

Pirson, M., and P.R. Lawrence. 2010. Humanism in business: Towards a paradigm shift? Journal of Business Ethics 93: 553-565.

Pless, N.M., T. Maak, and H. Harris. 2017. Art, ethics and the promotion of human dignity. Journal of Business Ethics 144: 223-232.

Putnam, L.L., A.M. Nicotera, and R. McPhee. 2009. Introduction: Communication constitutes organization. In Building theories of organization: The constitutive role of communication, ed. L.L. Putnam and A.M. Nicotera, 1-20. London: Routledge.

Rawls, John. 1971. A theory of justice. Oxford: Clarendon Press.

Ring, P.S. 1996. Fragile and resilient trust and their roles in economic exchange. Business \& Society 35 (2): $148-$ 175.

Rogers, K.M., and B.E. Ashforth. 2017. Respect in Organization: Feeling valued as "Me" and "We". Journal of Management 43: 1578-1608.

Rogers, K.M., K.G. Corley, and B.E. Ashforth. 2017. Seeing more than orange: Organizational respect and positive identity transformation in a prison context. Administrative Science Quarterly 62: 219-269.

Rosen, C. 2012. Dignity: Its history and meaning. Cambridge: Harvard University Press.

Rothausen, T.J., and K.E. Henderson. 2019. Meaning-based Job-Related Well-Being: Exploring a Meaningful Work Conceptualization of Job Satisfaction. Journal of Business and Psychology 34: 357-376.

Rothenbuhler, E. 1998. Ritual communication: From everyday conversation to mediated ceremony. Thousand Oaks: SAGE.

Ryan, R.M., and E.L. Deci. 2001. On happiness and human potentials:are view of research on hedonic and eudaimonic well-being. Annual Review of Psychology 52: 141-166.

Ryff, C., and C. Keyes. 1995. The structure of psychological well-being revisited. Journal of Personality and Social Psychology 69: 719-727.

Sen, A. 1999. Development as freedom. Oxford: Oxford University Press.

Seng, J.S., W.D. Lopez, M. Sperlich, L. Hamama, and C.D.R. Meldrum. 2012. Marginalized identities, discrimination burden, and mental health: Empirical exploration of an interpersonal-level approach to modeling intersectionality. Social Science \& Medicine 75 (12): 2437-2445.

Sharma, Neeru, and Paul G. Patterson. 1999. The impact of communication effectiveness and service quality on relationship commitment in consumer, professional services. Journal of Services Marketing 13 (2): 151-170.

Sison, A.J., I. Ferrero, and G. Guitian. 2016. Human dignity and the dignity of work: Insights from Catholic social teaching. Business Ethics Quarterly 26 (4): 503-528.

Solomon, Michael R., Carol Surprenant, John A. Czepiel, and Evelyn G. Gutman. 1985. A role theory perspective on dyadic interactions: the service encounter. Journal of Marketing 49 (1): 99-111. 
To, C.K.M., and K.K.B. Ko. 2016. Problematizing the collaboration process in a knowledge-development context. Journal of Business Research 69: 1604-1609.

United Nations 2018. https://www.un.org/sustainabledevelopment/. Accessed 7 Feb 2019.

Van der Have, R.P., and L. Rubalcaba. 2016. Social innovation research: An emerging area of innovation studies? Research Policy 45 (9): 1923-1935.

Vargo, S.L., and R.F. Lusch. 2008. Service-dominant logic: continuing the evolution. Journal of the Academy of Marketing Science 36 (1): 1-10.

Vargo, Stephen L., Paul P. Maglio, and Melissa Archpru Akaka. 2008. On value and value co-creation: A service systems and service logic perspective. European Management Journal 26 (3): 145-152.

Verhoef, Peter C. 2003. Understanding the effect of customer relationship management efforts on customer retention and customer share development. Journal of Marketing 67 (4): 30-45.

Wood, D.J. 1991. Corporate social performance revisited. Academy of Management Review 16 (4): 691-718.

Worline, Monica C., and Jane E. Dutton. 2017. Awakening Compassion at Work: The Quiet Power That Elevated People and Organizations. Oakland: Berrett-Koehler.

Publisher's Note Springer Nature remains neutral with regard to jurisdictional claims in published maps and institutional affiliations.

\title{
Affiliations
}

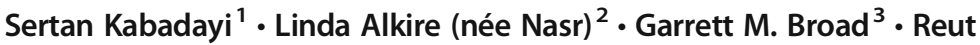 Livne-Tarandach $^{4} \cdot$ David Wasieleski $^{5,6} \cdot$ Ann Marie Puente $^{7}$}

\author{
Linda Alkire (née Nasr) \\ linda.alkire@txstate.edu \\ Garrett M. Broad \\ gbroad@fordham.edu \\ Reut Livne-Tarandach \\ livnere@gmail.com \\ David Wasieleski \\ wasieleski@duq.edu \\ Ann Marie Puente \\ annmariep@greyston.org
}

Gabelli School of Business, Fordham University, New York, New York, USA

2 Texas State University, San Marcos, TX, USA

3 Fordham University, The Bronx, NY, USA

4 The Management and Marketing Department, O’Malley School of Business, Manhattan College, New York, NY, USA

5 ICN Business School, Duquesne University, Pittsburgh, PA, USA

6 ICN Business School-ARTEM, Nancy-Metz, France

7 The Center for Open Hiring at Greyston, New York, NY, USA 